\title{
Gallbladder Cribriform Carcinoma
}

National Cancer Institute

\section{Source}

National Cancer Institute. Gallbladder Cribriform Carcinoma. NCI Thesaurus. Code C96891.

A rare variant of gallbladder adenocarcinoma. It is characterized by the presence of a malignant glandular epithelial infiltrate with a cribriform growth pattern. 